\title{
Mechanisms of Remodelling A Question of Life (Stem Cell Production) and Death (Myocyte Apoptosis)
}

\author{
Roberto Ferrari, $\mathrm{MD}, \mathrm{PhD}^{*}, * *$; Claudio Ceconi, $\mathrm{MD}, \mathrm{PhD}^{*}$; Gianluca Campo, $\mathrm{MD} *$; \\ Elisa Cangiano*; Caterina Cavazza*; \\ Paola Secchiero, $\mathrm{MD}, \mathrm{PhD}^{\dagger}$; Luigi Tavazzi, $\mathrm{MD}, \mathrm{PhD}^{\dagger \dagger}$
}

\begin{abstract}
Remodeling myocytes show a typical switch between the embryonic and classical features of apoptosis and/or hypertrophy representing a signal of death (ie, apoptosis) and a signal of life (ie, hypertrophy). The adult myocyte, however, is a terminal cell; usually it is unable to reproduce and death is not genetically programmed (apoptosis), but occurs by necrosis. The reinstatement of apoptosis and development of hypertrophy during remodeling could be part of the switch forward to the embryonic phenotype with reinstatement of the early embryonic genetic program. Hypertrophy and apoptosis are "sons" of the same "mother": the local, tissue neuroendocrine-neurohumoral response to a mechanical stretch of the myocytes consequent to the geometric changes imposed on the viable myocytes by the necrotic ones. As expected, the life and death cycle is very closely regulated by several autocrine systems, one of which is linked to the interleukin-6 family via a regulatory protein named GP-130. Activation of the GP-130 slows down the death signals, thus favoring hypertrophy and reducing fibrosis. (Circ J
\end{abstract} 2009; 73: 1973-1982)

Key Words: Apoptosis; Remodeling; Stem cells

$\mathbf{O}$ ur understanding of the molecular circuits at the basis of complex cardiac diseases such as myocardial ischemia and heart failure (HF) remains primitive. The challenge is to identify the genetic susceptibility, the environmental stress, as well as the modifiers of the disease, and to design new therapeutic strategies to interrupt the underlying pathological pathways.

However, because of a lack of surrogate models of disease onset and progression, new therapeutic strategies in cardiology today rely only on the crude endpoint of mortality in large-scale medical trials, which is why cardiovascular drug discovery is viewed as an extremely high-risk enterprise, particularly in the area of remodeling and HF. After the important recognition that the neuroendocrine response in $\mathrm{HF}$ is deleterious, rather than advantageous, no other fundamental steps have been made to understand the biology of the disease. Consequently, and not surprisingly, after the discovery of the usefulness of angiotensin-converting enzyme inhibitors (ACEI) and $\beta$-blockers for slowing down the progression of remodeling and HF, no real innovations have been made other than to identify a hypothetically "dangerous" hormone or cytokine to be blocked with a drug. Thus, cardiology has experienced several trials with antiangiotensin I receptor, anti-endothelins, anti-tumor necrosis factor (TNF)- $\alpha$ etc. All, unfortunately, provided not very exciting results.

Most likely, the field of cardiac biology is in transition: integrated genomic circuits that control myocyte survival, biochemical stress responses and cardiac contractility and electrical regulation are becoming available and a new classification of human cardiac disease can be envisaged in a short timeframe. ${ }^{1-3}$

We describe a different approach to the pathogenesis of cardiac remodeling, which considers the imbalance between myocyte death and survival, critical in both the progression of remodeling and the transition from left ventricular (LV) dysfunction to $\mathrm{HF}$.

\section{Cardiac Remodeling}

HF can no longer be considered a simple contractile disorder or disease of the heart alone. Clinical manifestations are, in fact, the result of changes to the heart's cellular and molecular components and to mediators that drive homeostatic control. There is general acceptance that as heart disease progresses into HF, heart size increases, cardiac function deteriorates and the symptoms of $\mathrm{HF}$ become evident. Although different terms have been used to describe it, cardiac remodeling encompasses many changes associated with progressive HF and it may be defined as "genome expression, molecular, cellular and interstitial changes that are manifested clinically as changes in size, shape and function of the heart after cardiac injury" ${ }^{4}$ The process of cardiac remodeling is influenced by hemo-

Received September 9, 2009; accepted September 11, 2009; released online October 10, 2009

*Chair of Cardiology, University Hospital of Ferrara, **Fondazione Salvatore Maugeri, IRCCS, †Sezione di Anatomia Umana University Hospital of Ferrara, Ferrara and ${ }^{\dagger}$ GVM Hospitals of Care and Research, Cotignola, Ravenna, Italy

Mailing address: Roberto Ferrari, MD, PhD, Chair of Cardiology, University Hospital of Ferrara, Corso Giovecca 203, 44100, Ferrara, Italy. E-mail: fri@unife.it

All rights are reserved to the Japanese Circulation Society. For permissions, please e-mail: cj@j-circ.or.jp 


\section{AMI MOBILIZES EPC STEM CELLS}

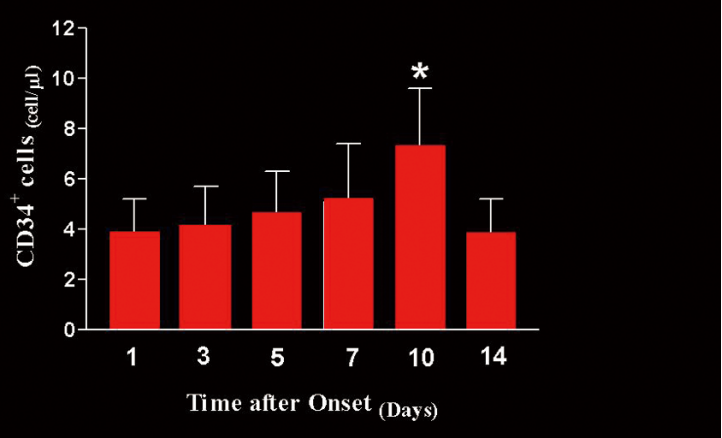

Figure 1. Acute myocardial infarction (AMI) increases the production and release of bone marrow stem cells and in particular, endothelial progenitor cells (EPC). Peak production is within 7-10 days after AMI.

dynamic load, neurohormonal activation and other factors still under investigation such as, for example, bone marrow stem cell production. The myocyte is the major cardiac cell involved in the remodeling process, but other components involved include the interstitium, fibroblasts, collagen and coronary vasculature; relevant processes also include ischemia, cell necrosis, hypertrophy and apoptosis. ${ }^{4}$

\section{When Does Remodeling Occur?}

Cardiac remodeling is as a physiologic and pathologic condition that may occur after myocardial infarction (MI), pressure overload (aortic stenosis, hypertension), inflammatory heart muscle disease (myocarditis), idiopathic dilated cardiomyopathy or volume overload (valvular regurgitation). Although the etiologies of these diseases are different, they share several pathways in terms of molecular, biochemical and mechanical events. Physiologic remodeling (ie, com- pensatory changes in the proportions and function of the heart) occurs in athletes, but will not be discussed further in this review because we are only addressing post-MI remodeling.

\section{Early Remodeling at the Necrotic Site}

After an infarct, the process of ventricular remodeling begins rapidly, usually within a few hours and continues to develop for almost a week. ${ }^{5,6}$ The process consists of cardiomyocyte lengthening, thinning of the infarct zone, followed by inflammation and eventually re-absorption of necrotic tissue, leading to scar formation. ${ }^{7-10}$ This phase of remodeling occurs at the site of the infarct and may, to some extent, be considered beneficial or adaptive. The myocyte becomes elongated as part of an initial compensatory process to maintain stroke volume after the loss of contractile tissue. In addition, the activation of both fibroblast and endothelial cells and the consequent collagen deposition contribute to scar formation, which undoubtedly should be considered a positive event, preventing heart rupture.

Although these series of events occur locally, at the site of the infarct, inflammation is a systemic response. Equally, the increased production and release from the bone marrow of stem cells, and in particular of endothelial progenitor cells (EPC) (Figure 1), is an intriguing and unexpected systemic response to the infarct. A peak of these cells in the blood occurs within 7-10 days after MI. It has been suggested that, at least in experimental models, the EPC show preferential homing within the necrotic area. The relative contribution of bone marrow EPC activation to the remodeling process is not clear. In theory, it could be an adaptive response because the EPC might contribute to angiogenesis in the necrotic or perinecrotic area. Interestingly, there is an inverse correlation between EPCs and with the degree of LV dysfunction: the higher the plasma level of these EPC, the better the ventricular function and vice versa. ${ }^{11,12}$ The precise role of stem cell regeneration in remodeling however, remains obscure.

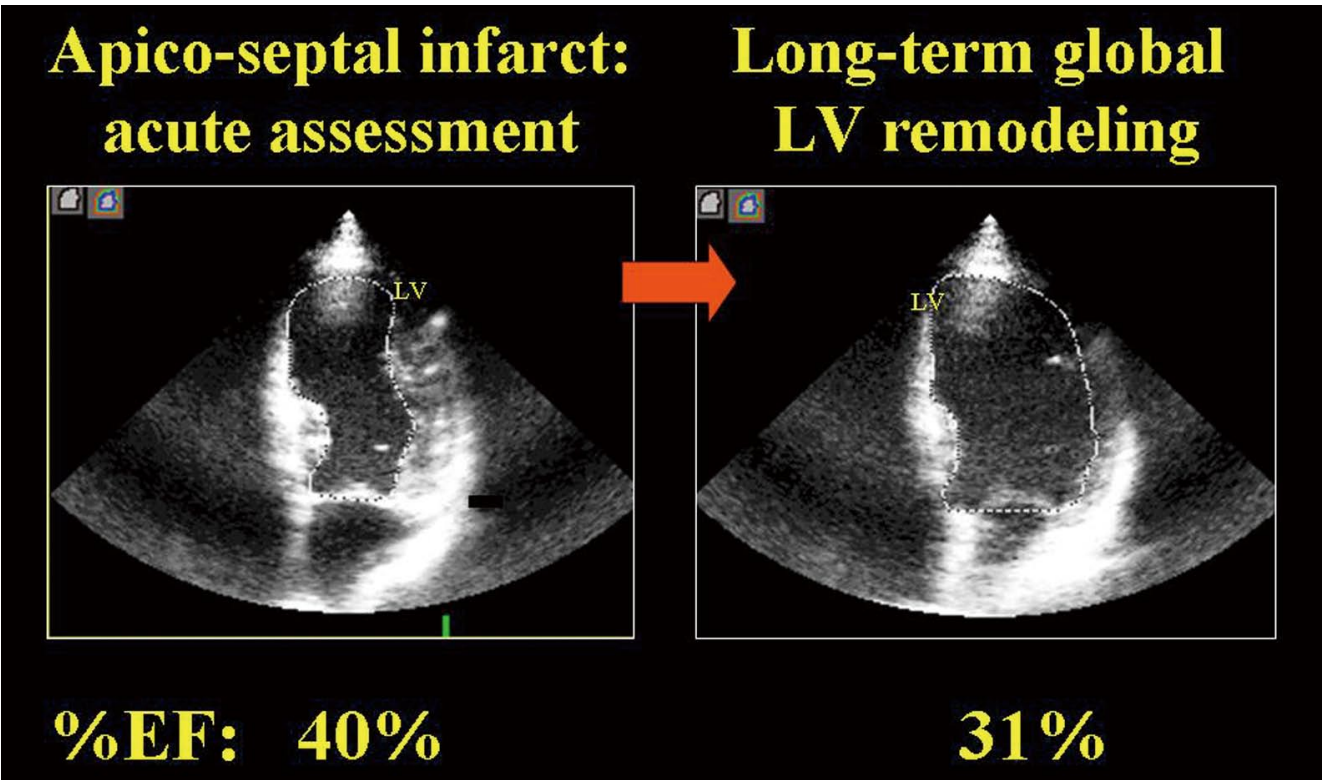

Figure 2. As the heart remodels, its geometry changes: it becomes less elliptical and more spherical. EF, ejection fraction; LV, left ventricular. 


\section{DEATH/SURVIVAL PATHWAYS}

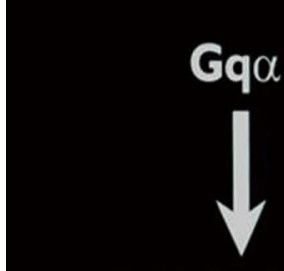

protein kinase

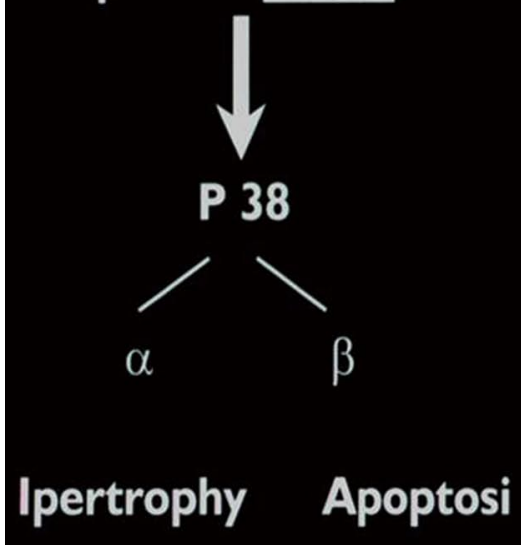

RAS

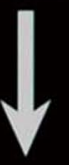

protein kinase

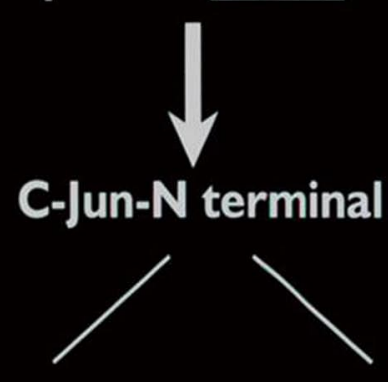

Figure 3. Death and survival paths have strong similarities. Although hypertrophy and apoptosis represent distinct phenotypic outcomes, the intracellular signals are very similar, involving the renin-angiotensin system (RAS), protein kinase paths, and components of MAP-kinase (p38 $\beta / \alpha$, Jun-N-terminal kinase), which have terminal signals that lead either to hypertrophy or to survival.

\section{Late Remodeling at Remote Sites}

Several studies of global LV chamber volumes and muscle mass show that the early dilatation (or early remodeling) might continue progressively, especially in elderly patients or in those with a large anterior wall MI. This late phase of remodeling ( $\geq 1$ month after infarct) occurs and involves sites at a distance from the infarcted area. As a result, the end-systolic volume index increases progressively and the ejection fraction declines. These are important predictors of mortality and remodeling could be seen as a bridge between LV dysfunction and HF. ${ }^{13,14}$

The importance of late remodeling as a pathogenic mechanism for HF is unclear. As the heart remodels, its geometry changes: it becomes less elliptical and more spherical (Figure 2). There are changes in ventricular mass, composition and volume, all of which may adversely effect cardiac function. 15,16 The actual changes occurring in the remote myocytes may be the major determinants of HF prognosis, rather than ventricular dilation itself. In fact, at the cellular level, the late phase of remodeling encompasses changes including myocyte hypertrophy, apoptosis, increased fibrillar collagen, fibroblast proliferation and eventually fibrosis. ${ }^{17-19}$ Interestingly, in cardiac biopsies from patients with HF undergoing cardiac transplantation or from experimental animals, the remodeled myocytes show a phenotype typical of embryonic life, with embryonic myofilaments, sarcoplasmic reticulum ATP-ase, and atrial natriuretic peptide in the ventricle, expression of ventricular funny current (if) channels and, more importantly, hypertrophic and apoptotic myocytes. ${ }^{20,21}$ These findings suggest that after an infarct there is a genomic shift toward a typical embryonic genome expression.

\section{Late Remodeling: Myocytes Return to Childhood}

The finding of both apoptotic and hypertrophied myocytes in remodeled myocardium is surprising: apoptosis is a clear expression of death whereas hypertrophy could be considered expression of life, particularly in the context of the heart, which normally comprises terminal cells that do not easily reproduce, although this dogma has recently been questioned.20

Several groups have tried to identify the precise signaling pathways that mediate these opposite responses: hypertrophy and apoptosis. The response is not immediate, as it is usually seen after months, or even years, following the infarct or the initial stimulus, which in animal and human models can be identified as mechanical cellular overload (eg, high blood pressure, valvular disease or post-MI).

In the late remodeled zone, in addition to hypertrophic or apoptotic myocytes, there are areas of fibrosis. Furthermore, the hypertrophic myocytes show features of a typical embryonic phenotype. Relevant to this discussion is the recognition that in embryonic life, myocytes undergo the life and death cycle: they die from apoptosis and do regenerate. Thus, in remodeling, there is a reinstatement of such a cycle. As in the embryonic myocytes, death occurs by apoptosis. Life or full regeneration, however, cannot occur completely, leading just to hypertrophy. This is probably a stereotypical cellular response to a biochemical/anatomical/ functional stress induced by the infarct, leading to hypertrophy and apoptosis. Interestingly, although hypertrophy and apoptosis represent distinct phenotypic outcomes, the intracellular signals are very similar, involving the renin-angiotensin system (RAS), Gp, and P38 $\beta / \alpha$ pathways (Figure 3).

A fundamental question is how a stressor that originates 


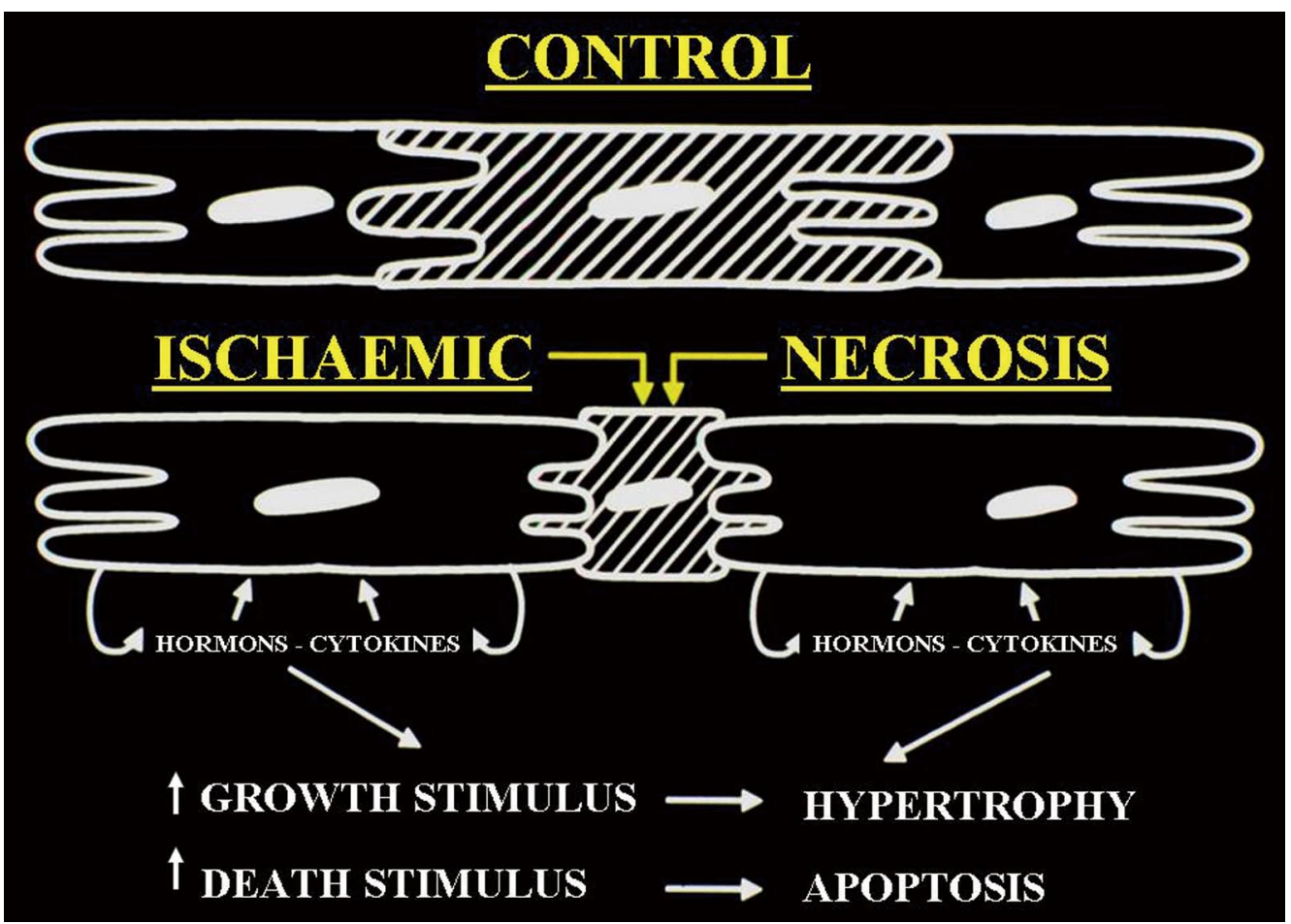

Figure 4. When myocytes die from ischemic necrosis, they undergo a drastic geometric change in their shape within a few seconds of oxygen deprivation. There is a sudden increase of intracellular calcium (hatched area), which causes hypercontraction of the myofilaments. These drastic mechanical changes, in turn, result in the stretch on other, still viable, myocytes. The stretch of the sarcolemma occurs at the level of the intercalated disc.

at the site of the infarct spreads to the remote, still viable myocytes.

\section{The Trigger for the Phenotype Switch}

During an infarct, myocytes die from necrosis, which is a very different type of death from apoptosis. Necrotic death is not programmed by the nuclei, but occurs in response to acute and prolonged ischemia. Necrotic death involves millions of myocytes at the same time, whereas apoptosis involves a single cell at a time. When myocytes die from necrosis, they undergo a drastic geometric change in their shape within few seconds of oxygen deprivation (Figure 4). From rectangular they become spherical. The necrotic myocyte shrinks because of the sudden increase in intracellular calcium, which causes hypercontraction of the myofilaments. These drastic mechanical changes, in turn, result in stretching of the sarcolemma of the other, still viable, myocytes.

In isolated myocytes, the mechanical stretch triggers in an autocrine manner, through the local release of hormone and cytokine growth and death signals, suggesting that myocytes are biochemical sensors. ${ }^{1,21}$ It is difficult to identify the precise mediators that induce the switch to the embryonic program, but likely candidates are angiotensin II, noradrenaline and aldosterone, at least judging by the documented capacity of ACEIs, $\beta$-blockers and anti-aldosterone drugs to reduce remodeling and improve survival in postMI patients. These and other hormones act extracellularly to induce intracellular signals that in turn are able to promote the nuclear changes ${ }^{21}$ (Figure 5).

It is therefore possible to envisage a series of events such as (1) myocytes dying as a result of the infarct, causing (2) a biochemical stretch transmitted to the remote, still viable myocytes, where in response, (3) hormones and cytokines are locally released and determine at the nuclear level a switch toward the fetal program, (4) reinstating the life and death cycle but without complete regeneration, leading to hypertrophy. (5) In the early phase, there is a matching between apoptosis and hypertrophy, but if apoptosis prevails, there is progressive myocyte loss with fibrotic substitution. (6) The hypertrophied and remaining myocytes show embryonic features, thus further contributing to pump failure and as a result, (7) the ventricle progressively enlarges, changes its shape and symptoms of HF progress.

\section{The Regulators of Progress}

All of the abovementioned steps are likely to be very closely regulated by many different systems, which under normal physiological conditions avoid this series of events from occurring. For instance, the adult heart develops different cell survival pathways, which inhibit myocyte apoptosis. ${ }^{22-25}$ Cardiotrophin-1, a Gp130 receptor-dependent cytokine, was isolated from mouse embryonic stem cell models of cardiogenesis and is a potent cardiac myocyte survival factor, able to suppress apoptosis. ${ }^{26}$ Several experimental studies support a cardiac protective effect of cardiotrophin-1 during hypoxic and other injuries. ${ }^{1}$ Equally, mice with a cardiac restricted abolition of cardiotrophin-1, and of gp130 function, cannot activate downstream survival pathways and display massive apoptosis of myocytes, acute $\mathrm{HF}$ and high mortality in response to moderate hypertension. ${ }^{27}$ Another paracrine factor, neuregulin, activates survival pathways and suppresses apoptosis in both ventricular and neuronal cells. Ablation of neuregulin or of its receptors 


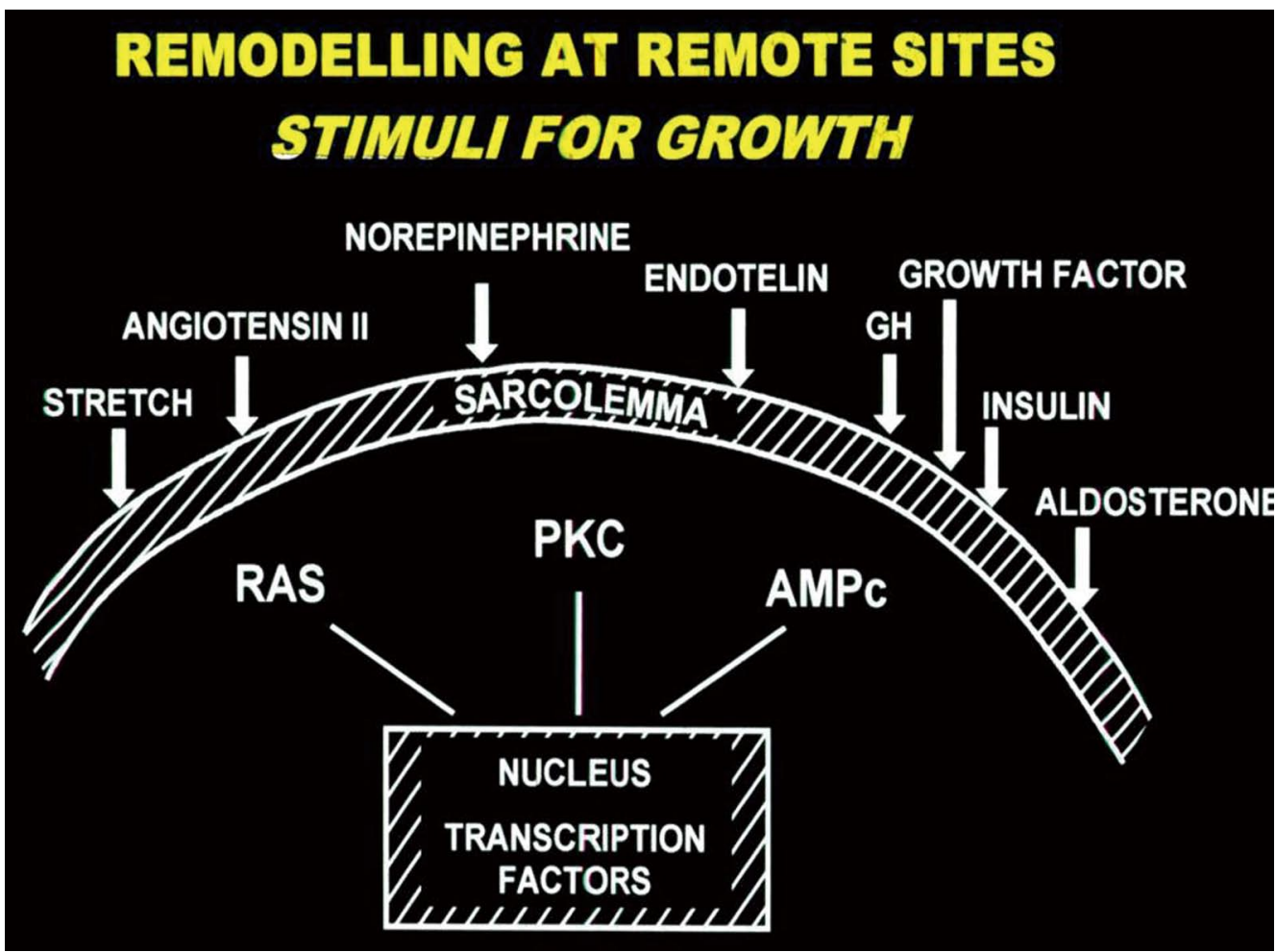

Figure 5. Nuclear changes leading to the embryonic program depend on signal systems such as those activated by reninangiotensin system (RAS), protein kinase C (PKC) and cyclic AMP (AMPc). Extracellular signals include angiotensin II, noradrenaline and aldosterone, as well as stretch.

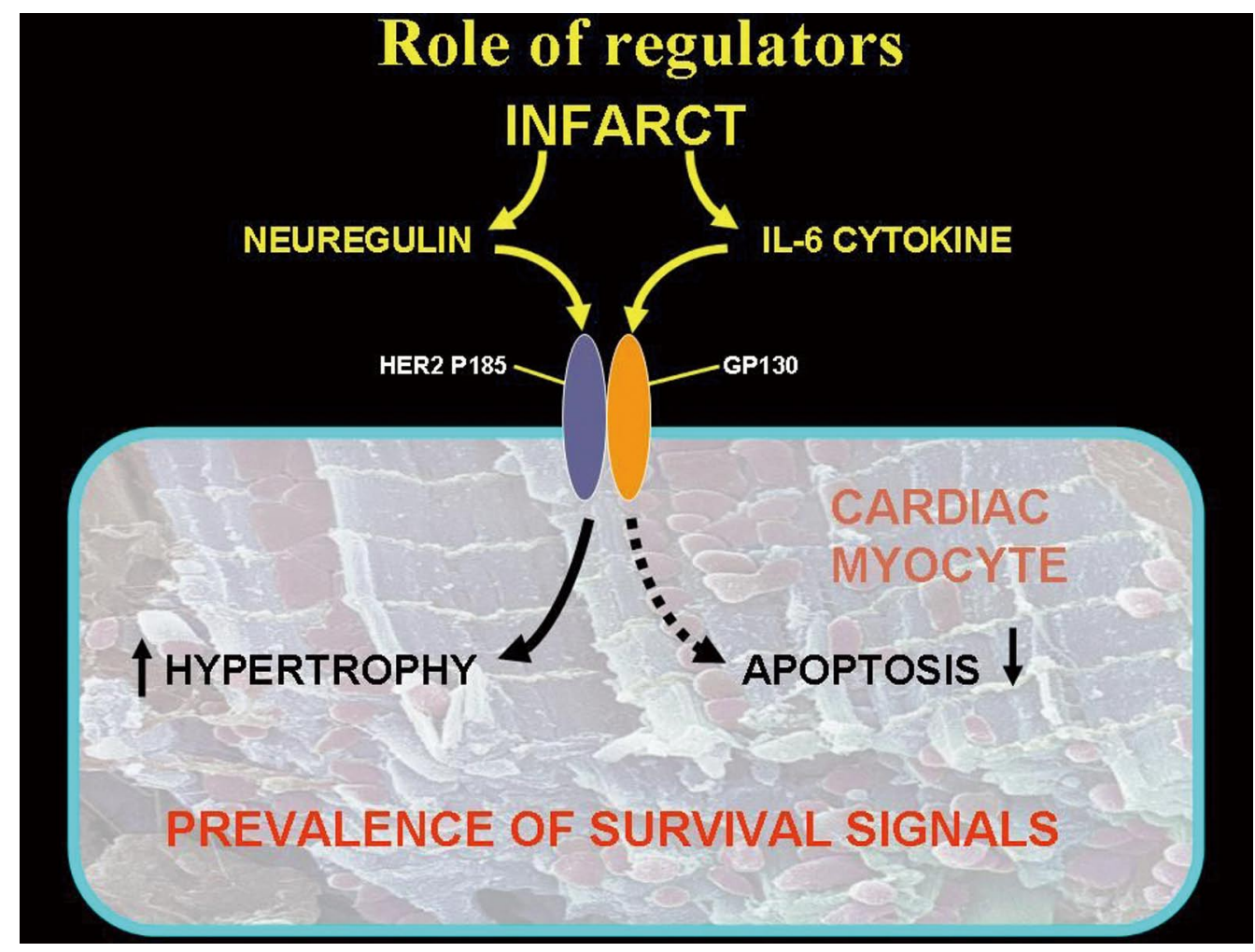

Figure 6. Factors leading to the prevalence of survival factors. Neuregulin is a paracrine factor that activates survival pathways and suppresses apoptosis in ventricular cells. Its receptors include gp185, which has a high affinity with gp130. Increased levels of the interleukin- 6 family cytokines, activated by myocardial infarction, stimulate gp130 to favor adaptive cardiac muscle hypertrophy and inhibit apoptosis, thus having beneficial effects. 


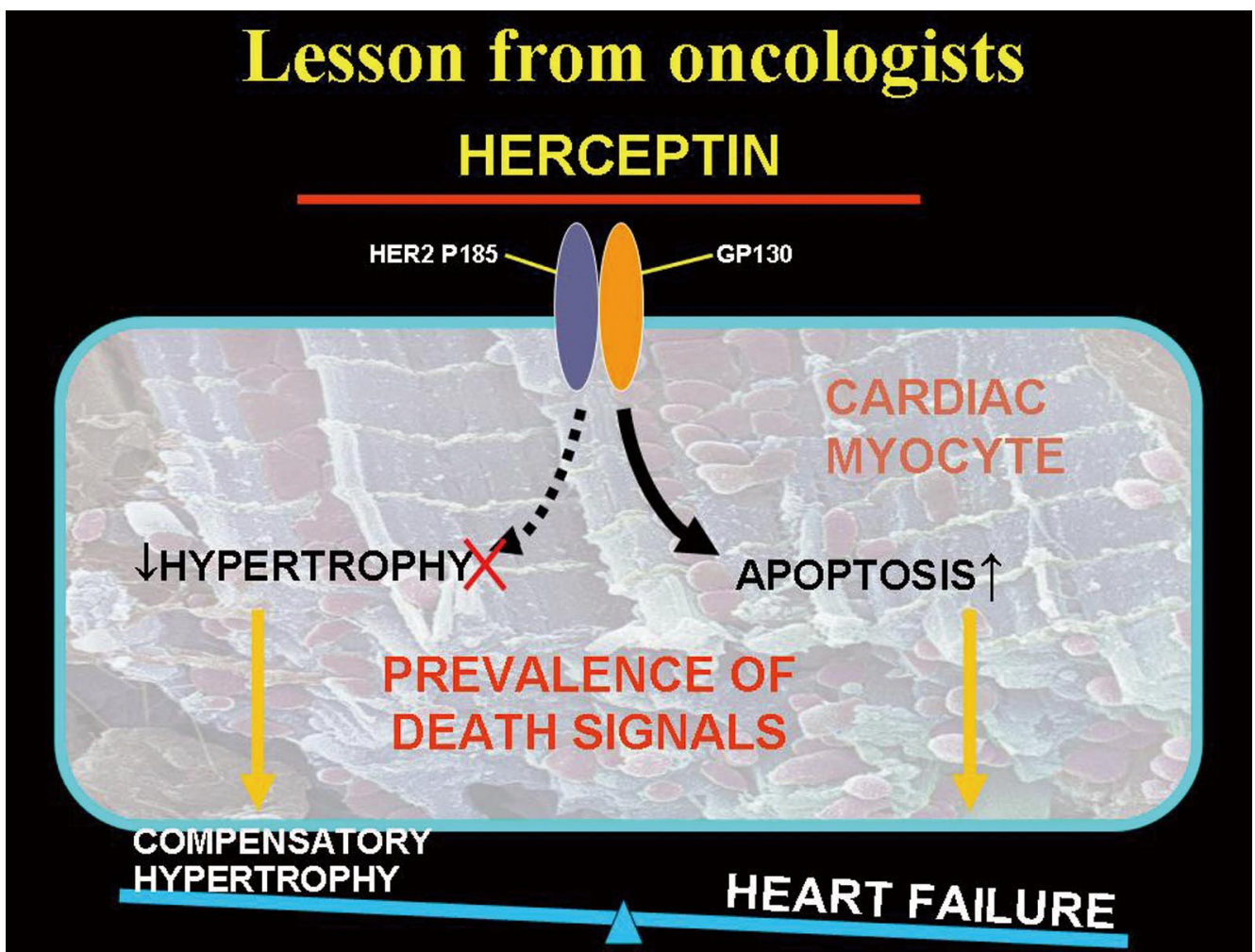

Figure 7. In unique translational research, oncologists have proved the importance of gp130 and pg 185 receptors in cardiac remodeling. The anti-tumor agent, trastuzumab (Herceptin), is used as an adjuvant therapy for ErbB2-positive cancer of the breast. It blocks the gp130 and pg185 receptors, and thereby reinstates apoptosis and the intracellular death signals, which help curb the cancer cells. However, trastuzumab also acts on the heart to block gp130 and pg185 receptors. The relatively incidental block of these because of cancer therapy reinstates apoptosis, and thus predisposes the patient to typical remodeling of the heart and thus heart failure.

ERB2 and ERB4 linked to gp185, which has a high affinity with gp130, also leads to oxidative stress and HF in the mouse embryo. Recent studies suggest that a new member of the interleukin (IL)- 6 cytokine family, CT-1, can activate several features of myocyte hypertrophy, including cell enlargement without proliferation, sarcomeric organization and embryonic gene expression. ${ }^{24-28}$ In addition, cardiotrophin-1 is capable of blocking the onset of cardiomyocyte apoptosis following stress. $24-28$

Finding that in remodeled myocardium there are apoptotic myocytes indicates a lack of cell survival pathways to inhibit the process and suggests a relevant role of gp130 and its analog gp185.

Role of gp130 and gp185: Lessons From Oncologists The role of gp130, and of its analog gp185, is evident from experiments in mice with ventricular restricted knockout of the gp130 gene and from clinical trials involving patients receiving anti-ERBB2 antibodies to treat metastatic breast cancer.

Although there is no apparent requirement for gp130 in the normal physiological heart, the protein is activated in response to pressure overload, and after an infarct, by an increased level of IL-6 family cytokines, which have pleiotropic effects on multiple organ systems, including the myocardium. The activation favors adaptive cardiac muscle hypertrophy and inhibits apoptosis, and thus has a beneficial effect (Figure 6). As a result, normal cardiac function can be maintained for a certain period, which explains why cardiomyopathy or overt HF after an infarct is usually seen only months or years later. However, the gp130 ventricular restricted knockout mice at 3-4 days after aortic banding or infarction already display a rapid onset of dilated cardiomyopathy accompanied by massive myocyte apoptosis, involving over $30 \%$ of the ventricular myocytes. ${ }^{1}$

Further evidence of the protective role of gp130 comes from patients with breast cancer undergoing treatment with trastuzumab, 29,30 which is an antibody able to block the gp130-gp185 and is used as an adjuvant therapy for ErbB2positive cancer breast because by blocking these receptors trastuzumab reinstates apoptosis and the intracellular death signals that are useful at the level of cancer cells. However, randomized clinical trials have shown that trastuzumab was unexpectedly associated with an increased incidence of HF, particularly when given together or after antraciline treatment. ${ }^{29,30}$ Most likely, antracliline by causing oxidative stress of the myocardium acts as a stressor to the heart. In response, as in after MI, the myocyte survival pathways mediated by the activation of gp130 are set into action. The relative incidental block of these by the cancer therapy reinstates apoptosis, and thus typical remodeling of the heart and HF (Figure 7).

This is a unique piece of translational research in which oncologists unexpectedly proved the importance of gp130 and gp185 in cardiac remodeling. 


\section{Late Remodeling: Balance Between Life and Death}

Taken together, these studies provide evidence that during late remodeling the myocyte survival pathways act to block the onset of myocyte apoptosis after a biochemical stress. Mechanical stimuli activate not only pathways that promote myocyte apoptosis, but concomitantly activate myocyte survival pathways such as hypertrophy. It is the failure of this tight control between the death and survival signals that most likely causes progression at the molecular level vs death and fibrosis, and at the clinical level progression vs overt HF. As often is the case in nature, it is a question of balancing opposing signals. Under normal circumstances, the myocyte survival pathways can effectively blunt the onset of death input in response to biochemical stress, leading to induction of a compensatory response. However, at a point late in time after an infarct, the balance tips such that there is a transition to dilated cardiomyopathy and HF.

It follows that inhibition of cardiac myocyte apoptosis is a valid target for the development of new therapeutic agents for cardiac HF. Equally, identification of downstream pathways (ie, gp130-, gp185-dependent ligands) that can promote cardiac myocyte survival is also of critical therapeutic interest. Finally, in such a contest where the death of myocytes prevails, it is obvious that stem cell transplantation could play an important therapeutic role.

\section{Influences on Cardiac Remodeling in the Clinical Setting}

\section{Changes in Hemodynamic Load}

Studies of global LV chamber volumes and muscle mass show that early LV dilation in patients with anterior MI may continue progressively and unabated; global compensatory (reactive) ventricular hypertrophy appears to be a delayed and limited adaptation during the first year. As a result, global LV wall tension and stresses increase considerably. The importance of remodeling as a pathogenic mechanism for $\mathrm{HF}$ is gaining consideration, and the factors leading to remodeling may be the major determinants of HF prognosis, rather than ventricular dilation itself. If cardiac dilation persists without hypertrophy, myocardial wall stress is increased creating a biochemical-molecular vicious circle and clinically this may lead to further dilation of the heart. Without therapy to reduce ventricular dilation, decrease wall stress and promote a favorable neurohormonal pattern, this process progresses towards overt chronic HF.

Neurohormonal activation in HF is known to mediate compensatory changes in response to falling cardiac output, but it is also a major component of disease progression and of the remodeling process. ${ }^{31-33}$

\section{Role of Catecholamines and $\boldsymbol{\beta}$-Blockers}

Plasma noradrenaline levels, reflecting increased adrenergic activation, are elevated in HF patients ${ }^{31,32}$ and are related to prognosis. ${ }^{31}$ Higher levels of circulating plasma noradrenaline correlate with a poorer long-term prognosis. ${ }^{31}$ Increased levels of catecholamines are deleterious because they increase heart rate, contractivity and peripheral resistance and thus myocardial oxygen consumption. At the molecular level, catecholamines promote the nuclear switch toward the embryonic phenotype and apoptosis as well as hypertrophy.

Multiple clinical trials have proved the benefits of $\beta$ - blockade in HF. In all those studies, $\beta$-blockers were administered in conjunction with an ACEI and diuretics. ${ }^{34-38}$

The $\beta$-blockade, in addition to ACE inhibition, consistently improves ejection fraction in both post-MI and HF patients, irrespective of etiology. ${ }^{34,38-43}$ Data from an echocardiographic substudy of the Australia/New Zealand (ANZ) Collaborative Group show that carvedilol significantly decreased the LV end-diastolic and end-systolic volume indexes and increased LV ejection fraction compared with placebo (ACE inhibition alone), suggesting a sustained improvement in cardiac remodeling. 39 These improvements were apparent by 6 months and maintained at 12 months $^{44,45}$ (Figure 3). Smaller scale studies have also shown that $\beta$-blockade with carvedilol ${ }^{39}$ and metoprolol ${ }^{36}$ has beneficial effects on LV geometry and mass (B 114, 129). An echocardiographic substudy from CIBIS I showed that after 5 months' treatment, LV fractional shortening increased and end-systolic dimensions were reduced significantly in the bisoprolol group compared with the placebo group; ${ }^{45}$ however, the LV end-diastolic dimensions did not change significantly with bisoprolol therapy. ${ }^{46}$

\section{Role of Inhibition of the RAS and of Its Inhibition}

In cell culture, angiotensin II increases DNA synthesis in myocardial fibroblasts and increases protein synthesis in both fibroblasts and myocytes. ${ }^{47}$ It appears to be an important mediator of the cellular responses to stretch, with local production resulting in proliferation and growth, and excessive angiotensin II causes oxidative stress with consequent induction of apoptosis at both the endothelial and myocyte level. ${ }^{48}$ Angiotensin II also increases coronary artery permeability, allowing diffusion of growth factors into the myocardial interstitium. ${ }^{15}$ It is known to cause necrosis and fibrosis through its cytotoxic effect on cardiac myocytes. ${ }^{49}$ Increased aldosterone production as a result of increased angiotensin II also has hemodynamic consequences and stimulates collagen synthesis by myocardial fibroblasts. ${ }^{50}$ Increased aldosterone levels may also play a role in myocyte death through their effect on electrolyte balance. ${ }^{51}$

The Survival and Ventricular Enlargement (SAVE), 52 Acute Infarction Ramipril Evaluation (AIRE), 53 and Trandolapril Cardiac Evaluation (TRACE) ${ }^{54}$ studies showed mortality benefits attributable to ACE inhibition in patients when treatment is started early after MI. A meta-analysis of ACEIs in post-MI patients concluded that early intervention (within 3 to 16 days of infarction) can slow the progression of cardiovascular disease and improve the survival rate. ${ }^{55}$ ACEIs also relieve symptoms and significantly improve survival in patients with HF compared with placebo. ${ }^{56-60}$ Interestingly, these agents favorably influence all parameters of LV remodeling in both asymptomatic LV dysfunction post-MI and symptomatic patients with HF.56,57,60,61 In patients with LV dysfunction, administration of captopril 1 week after Q-wave MI resulted in significant reductions in the LV end-diastolic and end-systolic volume indexes and significant increases in ejection fraction and the stroke volume index. In contrast, patients receiving furosemide or placebo showed significant increases in ventricular volumes, no change in the stroke volume index and reductions in ejection fraction. ${ }^{62}$ Using serial radionuclide ventriculograms, the SOLVD prevention trial showed that, compared with placebo, enalapril slowed or reversed LV dilation in patients with asymptomatic LV dysfunction but without clinical HF. ${ }^{58} \mathrm{LV}$ volumes were reduced to a lesser degree by enalapril treatment in asymptomatic compared with symptom- 


\section{TNF-a and EPC according to NYHA class}

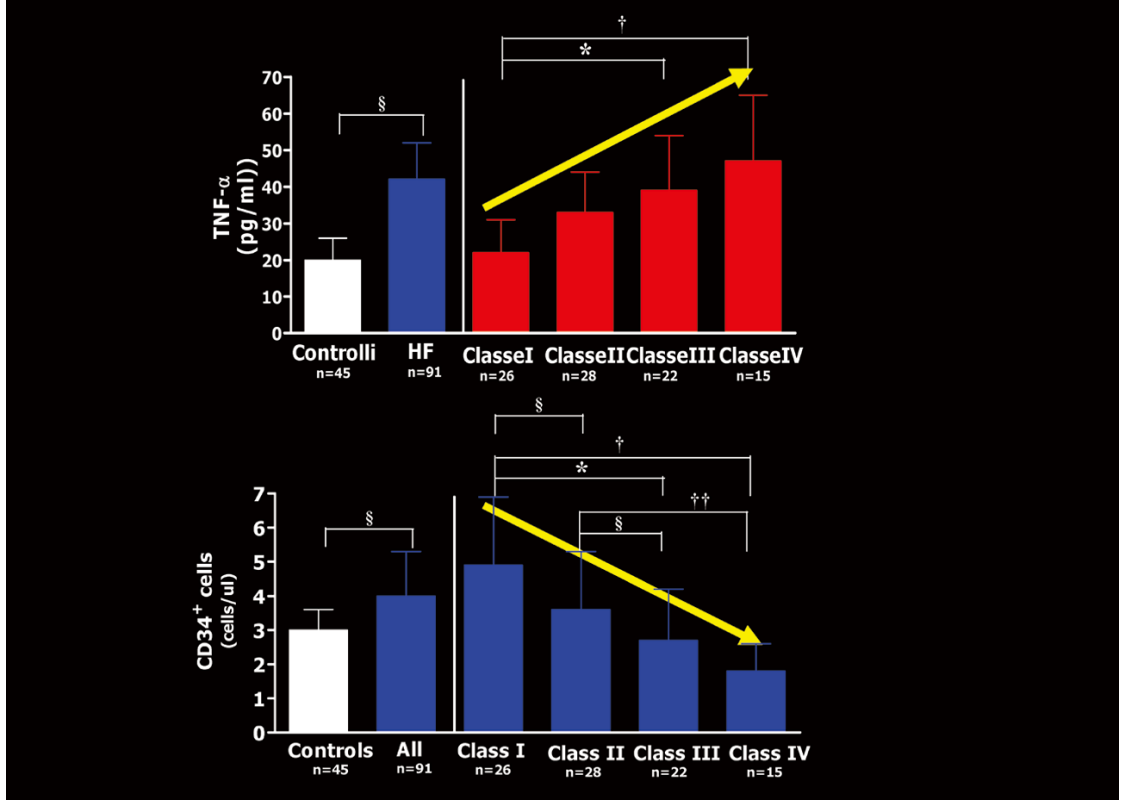

Figure 8. Tumor necrosis factor (TNF)- $\alpha$ and endothelial progenitor cells (EPC) according to New York Heart Association (NYHA) class. atic patients without HF. ${ }^{58}$ Although patient numbers were small, a SOLVD treatment substudy showed that chronic administration of enalapril prevented progressive LV dilation and systolic dysfunction compared with placebo in patients with mild to moderate HF and reduced the LV ejection fraction. ${ }^{57}$ The findings of these 2 studies were consolidated in a Doppler echocardiographic evaluation of 301 patients recruited from both the prevention and treatment arms of SOLVD, which showed that, compared with placebo, treatment with enalapril attenuated progressive increases in LV dilation and hypertrophy in patients with LV dysfunction, irrespective of the patient's symptomatic status.

\section{Role of LV Dysyncronization}

Cardiac resynchronization therapy (CRT) is an additional treatment for selected New York Heart Association (NYHA) class III and class IV patients with ventricular dyssynchrony. ${ }^{63,64}$ Multiple studies have documented improvements in survival, NYHA class, exercise capacity and quality of life. Interestingly, CRT also results in a significant decrease in LV size, assessed by LV end-diastolic and end-systolic diameters as early as after 1 month. There is a further progressive reduction in LV diameter and volume at 6 months, which is sustained at 1 year, provided that CRT is maintained. ${ }^{65,66}$ The time-dependent progressive reduction of remodeling (reverse remodeling) with CRT is associated with mechanically advantageous alterations in LV architecture, especially the reduction in mitral regurgitation. The intimate mechanisms of CRT-induced reverse remodeling are not known. Obviously, purely mechanical resynchronization is likely to play an important role. It has also been suggested that CRT is associated with favorable changes in established molecular markers of HF, including genes that regulate the shift toward the embryonic phenotype, contracting function as well as pathologic hypertrophy and apoptosis.

\section{Role of Aging}

A recent study, the Perindopril and Remodeling in Elderly with Acute Myocardial Infarction (PREAMI) has investigated an interesting group of patients: those who are older than 65 years, have survived an infarct and have normal LV function. ${ }^{67}$ The data show that in elderly post-MI patients, progressive LV remodeling can occur even in the presence of very small infarcts, which suggests that advanced age is an important determinant of LV remodeling. Despite normal pre-randomisation $\mathrm{LV}$ ejection fraction, the untreated elderly post-MI patients underwent progressive LV dilatation of a magnitude similar to that reported in other studies that enrolled younger patients, but with larger infarcted areas and greater LV dysfunction. ${ }^{67}$ Interestingly, administration of $8 \mathrm{mg}$ daily of perindopril for 12 months lead to a highly significant reduction in the LV remodeling in these elderly patients. The study does not provide any molecular explanation on why aging could cause remodeling, despite the occurrence of a very small infarct. It is known that aging favors apoptosis, so it may be possible that aging is a sort of "pre-conditioning" for post-remodeling. This may be relevant considering the increased aging of the population.

\section{Role of Stem Cells}

The role of bone marrow CD34+ cells and EPCs in patients with HF has been recently studied. ${ }^{12,68}$ In summary, the peripheral recruitment of CD34+ cells and EPCs is increased in the early stages of HF, whereas in class IV patients, their peripheral mobilization is reduced not only with respect to NYHA classes II and I but also with respect to controls, which yields an inverse correlation between $\mathrm{CD}^{+}{ }^{+}$cells and the severity of the disease (Figure 8).

Our previous work has shown that incubation of serum from HF patients induces endothelial cell apoptosis and endothelial nitric oxide synthase downregulation, ${ }^{69}$ which explains the severe endothelial dysfunction in this setting. Therefore, it is tempting to speculate that increased levels of $\mathrm{CD} 4^{+}$and EPCs during $\mathrm{HF}$ may be a reflection of 
diffuse and severe endothelial damage.

The inverse correlation between CD34+ cells, TNF- $\alpha$ and related soluble receptors points to a role of TNF- $\alpha$ in this biphasic response. During the early stages of the disease, when TNF- $\alpha$ is not yet significantly elevated, CD $34^{+}$cells and EPCs are increased, as a reflection of a functional bone marrow response to diffuse and severe endothelial damage. In advanced disease phases, an additional and significant increase in TNF- $\alpha$ occurs, which, by exerting a suppressive effect on hemopoiesis, ${ }^{70}$ finally counteracts and overwhelms the triggers able to increase $\mathrm{CD} 34^{+}$and EPC mobilization during the early phases (Figure 8).

\section{Closing Remarks}

There is little doubt that remodeling and its role in disease progression are multimechanistic and complex. Post-MI remodeling is clinically relevant and will increase in relevance with the increasing age of the population. Symptoms need not be the exclusive guide as to when therapy should be initiated, and the choice of therapy should take into account all the underlying components that contribute to disease progression, with remodeling as a critical component. The available molecular explanations for the process would suggest that therapy should be implemented as early as possible. The key next steps will be the determination of how the information generated from cellular and molecular models can be used, together with data from clinical trials, to ensure that patients receive optimal therapy at an appropriate time to slow disease progression. Reversing remodeling and preventing further remodeling is one way of slowing disease progression. ACE inhibition, $\beta$-blockade and CRT, which reduce morbidity and mortality and improve a number of remodeling parameters, may offer such a therapeutic approach. The challenge is to develop new and more specific treatments that may be even more effective in reversing the structural abnormalities in the left ventricle. Administration of appropriate stem cells could be a possibility.

\section{Acknowledgments}

The authors thank Dr Monia Monti and Dr Stefania Gambetti (Medical Trials Analysis, Ferrara, Italy) for their assistance in collecting the data and performing the laboratory tests. The study was supported by RegionalUniversity project of Emilia Romagna-Area IB - Innovative Research.

\section{References}

1. Chien K. Genomic circuits and the interactive biology of cardiac diseases. Nature 2000; 407: 227-232.

2. Chien KR. Stress pathways and heart failure. Cell 1999; 98: 555558.

3. Zhao YY, Sawyer DR, Baliga RR, Opel DJ, Han X, Marchionni MA, et al. Neuregulins promote survival and growth of cardiac myocytes: Persistence of ErbB2 and ErbB4 expression in neonatal and adult ventricular myocytes. J Biol Chem 1998; 273: 10261-10269.

4. Cohn JN, Ferrari R, Sharpe N. Cardiac remodeling: Concepts and clinical implications: A consensus paper from an international forum on cardiac remodelling. J Am Coll Cardiol 2000; 35: 269-282.

5. Eaton LW, Bulkley BH. Expansion of acute myocardial infraction: Its relationship to infarct morphology in a canine model. Circ Res 1981; 49: 80-88.

6. Hochman JS, Bulkley BH. Expansion of acute myocardial infarction; an experimental study. Circulation 1982; 65: 1446-1450.

7. Weisman H, Bush DE, Mannisi JA, Bulkley BH. Global cardiac remodeling after acute myocardial infarction: A study in the rat model. Am J Cardiol 1985; 5: 1355-1362.

8. McKay RG, Pfeffer MA, Pasternak RC, Markis JE, Come PC, Nakao S. Left ventricular remodeling after myocardial infarction: A corollary to infarct expansion. Circulation 1986; 74: 693-702.
9. Hutchins GM, Bulkley BH. Infarct expansion extension: Two different complications of acute myocardial infarction. Am J Cardiol 1978; 41: $1127-1133$.

10. Anversa P, Olivetti G, Capasso JM. Cellular basis of ventricular remodeling. JACC 2000; 35: 569-582.

11. Valgimigli M, Rigolin GM, Cittanti C, Malagutti P, Curello S, Percoco GF, et al. Use of granulocyte-colony stimulating factor during acute myocardial infarction to enhance bone marrow stem cell mobilization in humans: Clinical and angiographic safety profile. Eur Heart J 2005; 26: $1838-1845$.

12. Valgimigli M, Rigolin GM, Fucili A, Della Porta M, Soukhomovskaia $\mathrm{O}$, Malagutti $\mathrm{P}$, et al. CD34+ and endothelial progenitor cells in patients with various degrees of congestive heart failure. Circulation 2004; 110: 1209-1212.

13. Gaudron P, Eilles C, Kugler I, Ertl G. Progressive left ventricular dysfunction and remodeling after myocardial infarction. Circulation 1993; 87: 755-763.

14. White HD, Norris RM, Brown MA, Brandt PWT, Whitlock RML, Wild CJ. Left ventricular end-systolic volume as the major determinant of survival after recovery from endomyocardial infarction. Circulation 1987; 76: $44-51$.

15. Weber KT, Brilla CG. Pathological hypertrophy and the cardiac interstitium: Fibrosis and the renin-angiotensin-aldosterone system. Circulation 1991; 83: 1849-1865.

16. Struijker-Boudier HAJ, Smits JFM, DeMey JGR. Pharmacology of cardiac and vascular remodeling. Anпи Rev Pharmacol Toxicol 1995; 35: $509-539$.

17. Olivetti G, Abbi R, Quaini F, Kajstura J, Cheng W, Nitahara JA. Apoptosis in the failing human heart. N Engl J Med 1997; 336: $1131-1141$.

18. Anderson KR, Sutton MG, Lie JT. Histopathological types of cardiac fibrosis in myocardial disease. J Pathol 1978; 128: 79-85.

19. Villareal FJ, Kim NN, Ungab GD, Printz MP, Dillmann WH. Identification of functional angiotensin II receptors on rat cardiac fibroblasts. Circulation 1993; 88: 2849-2861.

20. Kajstura J, Leri A, Finato N, di Loreto C, Bertrami CA, Anversa P. Myocyte proliferation in end stage cardiac failure in humans. Proc Natl Acad Sci USA 1998; 95: 8801-8805.

21. Hirota H, Chen J, Betz UA, Rajewsky K, Gu Y, Ross J Jr, et al. Loss of a gp130 cardiac muscle cell survival pathway is a critical event in the onset of heart failure during biomechanical stress. Cell 1999; 97: $189-198$.

22. Meyer D, Birchmeier C. Multiple essential functions of neuregulin in development. Nature 1995; 378: 386-390.

23. Sheng Z, Pennica D, Wood WI, Chien KR. Cardiotrophin-1 displays early expression in the murine heart tube and promotes cardiac myocyte survival. Development 1996; 122: 419-428.

24. Wollert KC, Chien KR. Cardiotrophin-1 and the role gp130-dependent signaling pathways in cardiac growth and development. $J \mathrm{Mol}$ Med 1997; 75: 492-501.

25. Patel PA, Tilley DG, Rockman HA. Beta-arrestin-mediated signaling in the heart. Circ J 2008; 72: 1725-1729.

26. Taga T, Kishimoto T. Gp130 and the interleukin-6 family of cytokines. Annu Rev Immunol 1997; 15: 797-819.

27. Teiger E, Than VD, Richard L, Wisnewsky C, Tea BS, Gaboury L, et al. Apoptosis in pressure overload-induced heart hypertrophy in the rat. J Clin Invest 1996; 97: 2891-2897.

28. Wang Y, Huang S, Sah VP, Ross J Jr, Brown JH, Han J, et al. Cardiac muscle cell hypertrophy and apoptosis induced by distinct members of the p38 mitogen-activated protein kinase family. J Biol Chem 1998; 273: 2161-2168.

29. Perik PJ, de Korte MA, van Veldhuisen DJ, Gietema JA, Sleijfer DT, de Vries EG. Cardiotoxicity associated with the use of trastuzumab in breast cancer patients. Expert Rev Anticancer Ther 2007; 7: 1763 1771.

30. Ewer MS, Lippman SM. Type II chemotherapy-related cardiac dysfunction: Time to recognize a new entity. J Clin Oncol 2006; 23: $2900-2902$.

31. Cohn JN, Levine TB, Olivari MT, Garberg V, Lura D, Francis GS, et al. Plasma norepinephrine as a guide to prognosis in patients with chronic congestive heart failure. N Engl J Med 1984; 311: 819-823.

32. Chidsey C, Braunwald E, Morrow AG, Mason DT. Myocardial norepinephrine concentrations in man. N Engl J Med 1963; 269: 653658.

33. McClure S, Carunana L, Davie AP, Goldthorp S, McMurray JJ. Cohort study of plasma natriuretic peptides for identifying left ventricular systolic dysfunction in primary care. BMJ 1998; 317: 516519.

34. Waagstein F, Bristow MR, Swedberg K, Camenini F, Fowler MB, Silver MA. Beneficial effects of metoprolol in idiopathic dilated car- 
diomyopathy. Lancet 1993; 342: 1441-1446.

35. Packer M, Bristow MR, Cohn JN, for the US Carvedilol Heart Failure Study Group. The effect of carvedilol on morbidity and mortality in patients with chronic heart failure. N Engl J Med 1996; 334: $1349-1355$.

36. MERIT-HF Study Group. Effect of metoprolol CR/XL in chronic heart failure: Metoprolol CR/XL randomized intervention trial in congestive heart failure. Lancet 1999; 353: 2001-2007.

37. CIBIS II Investigators and Committees. The Cardiac Insufficiency Bisoprolol Study II (CIBIS II): A randomized trial. Lancet 1999; 353: $9-13$.

38. CIBIS Investigators and Committee. A randomized trial of betablockade in heart failure: The Cardiac Insufficiency Bisoprolol Study (CIBIS). Circulation 1994; 90: 1765-1773.

39. Australia/New Zealand Heart Failure Research Collaborative Group. Randomized, placebo-controlled trial of carvedilol in patients with congestive heart failure due to ischemic heart disease. Lancet 1997; 349: $375-380$.

40. Bristow MR, Gilbert EM, Abraham WT, Adams KF, Fowler MB, Hershberger RE, et al; MOCHA investigators. Carvedilol produces dose-related improvements in left ventricular function and survival in subjects with chronic heart failure. Circulation 1996; 94: 2807-2816.

41. Basu S, Senior R, Raval U, van der Does R, Bruckner T, Lahiri A. Beneficial effects of intravenous and oral carvedilol treatment in acute myocardial infarction: A placebo-controlled randomized trial. Circulation 1997; 96: 183-191.

42. Packer M, Colucci WS, Sackner-Bernstein JD, Liang CS, Goldscher DA, Freeman I, et al. Double-blind, placebo-controlled study of the effects of carvedilol in patients with moderate to severe heart failure: The PRECISE trial [Prospective Randomized Evaluation of Carvedilol on Symptoms and Exercise]. Circulation 1996; 94: 2793-2799.

43. Cohn JN, Fowler MB, Bristow MR, Colucci WS, Gilbert EM, Kinhal $\mathrm{V}$, et al. Safety and efficacy of carvedilol in severe heart failure: The US Carvedilol Heart Failure Study Group. J Card Fail 1997; 3: $173-179$.

44. Doughty RN, Whalley GA, Gamble G, MacMahon S, Sharpe N. Left ventricular remodeling with carvedilol in patients with congestive heart failure due to ischemic heart disease. J Am Coll Cardiol 1997; 29: $1060-1066$

45. Greenberg B, Quinones MA, Koilpillai C, Limacher M, Shindler D, Benedict C, et al. Effect of long-term enalapril on cardiac structure and function in patients with left ventricular dysfunction. Circulation 1995; 91: 2573-2581.

46. Lechat P, Escolano S, Golmard JL. Prognostic value of bisoprololinduced hemodynamic effects in heart failure during the Cardiac Insufficiency Bisoprolol Study (CIBIS). Circulation 1997; 96: 2197 2205.

47. Sadoshima J, Izumo S. Molecular characterization of angiotensin IIinduced hypertrophy of cardiac myocytes and hyperplasia of cardiac fibroblasts: Critical role of the AT1 receptor subtype. Circ Res 1993; 73: $413-423$.

48. Sadoshima J, Xu Y, Slayter HS, Izumo S. Autocrine release of angiotensin II mediates stretch-induced hypertrophy of cardiac myocytes in vivo. Cell 1993; 75: 977-984.

49. Ollivier JP, Bouchet VA. Prospects for cardioreparation. Am J Cardiol 1992; 70: $27-36 \mathrm{C}$

50. Brilla CG, Zhou G, Matsubara L, Weber KT. Collagen metabolism in cultured adult rat cardiac fibroblasts: Response to angiotensin II and aldosterone. J Mol Cell Immunol 1994; 26: 809-820.

51. Sheehan JP, Seelig MS. Interactions of magnesium and potassium in the pathogenesis of cardiovascular disease. Magnesium 1984; 3: 301-314.

52. Pfeffer MA, Braunwald E, Moyé LA, Basta L, Brown EJ Jr, Cuddy TE, et al. Effect of captopril on mortality and morbidity in patients with left ventricular dysfunction after myocardial infarction: Results of the Survival and Ventricular Enlargement Trial. N Engl J Med 1992; 327: 669-677.

53. The Acute Infarction Ramipril Efficacy (AIRE) Study Investigators. Effect of ramipril on mortality and morbidity of survivors of acute myocardial infarction with clinical evidence of heart failure. Lancet
1993; 342: 821-828.

54. Køber L, Torp-Pedersen C, Carlsen JE, Bagger H, Eliasen P, Lyngborg $\mathrm{K}$, et al. A clinical trial of the angiotensin-converting enzyme inhibitor trandolapril in patients with left ventricular dysfunction after myocardial infarction: Trandolapril Cardiac Evaluation (TRACE) Study Group. N Engl J Med 1995; 333: 1670-1676.

55. Beckwith C, Munger MA. Effect of angiotensin-converting enzyme inhibitors on ventricular remodeling and survival following myocardial infarction. Ann Pharmacother 1993; 27: 755-766.

56. Cohn JN, Johnson G, Ziesche S, Cobb F, Francis G, Tristani F, et al. A comparison of enalapril with hydralazine-isosorbide dinitrate in the treatment of chronic congestive heart failure. N Engl J Med 1991; 325: $303-310$.

57. The SOLVD Investigators. The effect of enalapril on survival in patients with reduced left ventricular ejection fraction and congestive heart failure. N Engl J Med 1991; 325: 293-302.

58. Garg R, Yusuf S. Overview of randomized trials of angiotensin-converting enzyme inhibitors on morbidity and mortality in patients with heart failure: Collaborative Group on ACE Inhibitor Trials. JAMA 1995; 273: 1450-1456.

59. Iraqi W, Rossignol P, Angioi M, Fay R, Nuée J, Ketelslegers JM, et al. Extracellular cardiac matrix biomarkers in patients with acute myocardial infarction complicated by left ventricular dysfunction and heart failure: Insights From the Eplerenone Post-Acute Myocardial Infarction Heart Failure Efficacy and Survival Study (EPHESUS) Study. Circulation 2009; 119: 2471-2479.

60. The CONSENSUS Trial Study Group. Effects of enalapril on mortality in severe congestive heart failure: Results of the Cooperative North Scandinavian Enalapril Survival Study (CONSENSUS). N Engl J Med 1987; 316: 1429-1435.

61. Pfeffer MA, Lamas GA, Vaughan DE, Parisi AF, Braunwald E. Effect of captopril on progressive ventricular dilation after anterior myocardial infarction. N Engl J Med 1987; 317: 787-792.

62. Sharpe N, Murphy J, Smith H, Hannan S. Treatment of patients with symptomless left ventricular dysfunction after myocardial infarction. Lancet 1988; 1: 253-259.

63. Sutton M St John, Keane MG. Reverse remodelling in heart failure with cardiac resynchronisation therapy. Heart 2007; 93: 167-171.

64. Chakir K, Daya SK, Aiba T, Tunin RS, Dimaano VL, Abraham TP, et al. Mechanisms of enhanced beta-adrenergic reserve from cardiac resynchronization therapy. Circulation 2009; 119: 1231-1240.

65. Chuang ML, Hibberd MG, Salton CJ, Beaudin RA, Riley MF, Parker RA, et al. Importance of imaging method over imaging modality in noninvasive determination of left ventricular volumes and ejection fraction: Assessment by two- and three-dimensional echocardiography and magnetic resonance imaging. J Am Coll Cardiol 2000; 35: $477-484$.

66. St John Sutton M, Pfeffer MA, Plappert T, Rouleau JL, Moyé LA, Dagenais GR, et al. Quantitative two-dimensional echocardiographic measurements are major predictors of adverse cardiovascular events after acute myocardial infarction: The protective effects of captopril. Circulation 1994; 89: 68-75.

67. PEAMI Investigators. Effects of angiotensin-converting enzyme inhibition with perindopril on left ventricular remodelling and clinical outcome: Results of the PREAMI Study. Arch Intern Med 2006; 166: 659-666.

68. Yip HK, Chang LT, Wu CJ, Sheu JJ, Youssef AA, Pei SN, et al. Autologous bone marrow-derived mononuclear cell therapy prevents the damage of viable myocardium and improves rat heart function following acute anterior myocardial infarction. Circ J 2008; 72: 13361345 .

69. Agnoletti L, Curello S, Bachetti T, Malacarne F, Gaia G, Comini L, et al. Serum from patients with severe heart failure downregulates eNOS and is proapoptotic: Role of tumor necrosis factor- $\alpha$. Circulation 1999; 100: 1983-1991.

70. Dufour C, Corcione A, Svahn J, Haupt R, Poggi V, Béka'ssy AN, et al. TNF- $\alpha$ and IFN- $y$ are overexpressed in the bone marrow of Fanconi anaemia patients and TNF- $\alpha$ suppresses erythropoiesis in vitro. Blood 2003; 102: 2053-2059. 